\title{
Turismo de compras: Retos, oportunidades y estrategias de desarrollo para el destino La Habana.
}

Shopping tourism: challenges, opportunities and development strategies for the destination Havana.

\author{
Msc. Danay M. Bulnes Mann. ${ }^{1}$
}

\begin{abstract}
In recent years, the relationship between trade and tourism has grown stronger, and consequently, the modality of shopping tourism has developed even more. The present research aims to identify the challenges and opportunities of the Havana destination for the development of the shopping tourism modality, based on the study of the market (demand and competition) and the supply of the destination. To fulfill this objective, a benchmarking was carried out between various shopping destinations in Latin America and Havana, and the attractiveness-competitiveness-tourism modality matrix was applied for various demand segments. The study was divided into several moments, firstly, a conceptualization was carried out on the modality of shopping tourism; in a second moment, the modality for its development in the Havana destination was assessed, based on the trade of goods, benchmarking and the matrix; and the investigation was completed with the determination of challenges, opportunities and development strategies for the modality in this destination. As scientific basis for the research, empirical, theoretical and statistical methods were used, such as the interview, non-participatory observation, documentary analysis and comparative analysis.
\end{abstract}

Key Words: Shopping Tourism, Benchmarking, Matrix Of AttractivityCompetitiveness-Tourist Modality.

\section{Resumen}

En los últimos años ha tomado mayor fuerza la relación entre el comercio y el turismo, y en consecuencia, se ha desarrollado aún más la modalidad de turismo de compras. La presente investigación tiene como objetivo identificar los retos y oportunidades del destino La Habana para el desarrollo de la modalidad de turismo de compras, a partir del estudio del mercado (demanda y competencia) y de la oferta del destino. Para dar

\footnotetext{
${ }^{1}$ Profesora de la Facultad de Turismo. Universidad de La Habana, Cuba, danay_bulnes@ftur.uh.cu
} 
cumplimiento a este objetivo, se llevó a cabo un benchmarking entre varios destinos de compras en América Latina y La Habana, y se aplicó la matriz de atractividadcompetitividad-modalidad turística para varios segmentos de demanda. El estudio se dividió en varios momentos, primeramente, se realizó una conceptualización sobre la modalidad de turismo de compras; en un segundo momento se valoró la modalidad para su desarrollo en el destino La Habana, a partir del comercio de bienes, el benchmarking y la matriz; y se culminó la investigación con la determinación de retos, oportunidades y estrategias de desarrollo para la modalidad en este destino. Como base científica de la investigación se emplearon métodos empíricos, teóricos y estadísticos, tales como la entrevista, la observación no participativa, el análisis documental y el análisis comparativo.

Palabras Clave: Turismo De Compras, Benchmarking, Matriz De AtractividadCompetitividad-Modalidad Turística.

\section{Introducción}

En la actualidad, el turismo es uno de los sectores más lucrativos para las economías, tanto así que en muchos países constituye la principal fuente de ingresos. Como fenómeno, ha experimentado un continuo crecimiento debido al incremento de la diversificación y de la competencia entre los destinos. Además, constituye uno de los principales actores del comercio internacional.

$\mathrm{Al}$ cierre del año 2018 las llegadas de turistas internacionales crecieron un 6\%, llegando a los 1400 millones según el Barómetro Mundial de Turismo de la Organización Mundial del Turismo (en lo adelante OMT). El pronóstico a largo plazo de la OMT publicado en 2010 indicaba que se alcanzarían los 1400 millones en 2020, pero con el progresivo crecimiento de las llegadas internacionales, dicho pronóstico se ha adelantado dos años. (OMT, 2019) A propósito, el actual secretario general de la OMT Zurab Pololikashvili, expresó: "Los viajes internacionales siguen creciendo con fuerza, consolidando al sector turístico como un motor clave del desarrollo económico. Como tercer sector ex portador del mundo, el turismo es esencial para la creación de empleo y la prosperidad de las comunidades" (Organización Mundial del Turismo, 2018). De esa forma, es fácil comprender el papel prioritario que esta actividad ostenta en un gran número de países.

Cuba es uno de los países que ha apostado por el turismo como sector estratégico e impulsor de su economía. Ejemplo de ello ha sido el crecimiento sostenido por encima del 10\% en el arribo de turistas internacionales a la Isla en los últimos 3 años (Hosteltur, 2018). Según las estadísticas de la Oficina Nacional de Estadística e Información (en lo adelante ONEI), en el 2018 Cuba alcanzó la cifra total de 4711910 visitantes internacionales, superando en 58351 el año anterior. Manteniendo como principales mercados emisores a Canadá en primer lugar, Estados Unidos y la Comunidad Cubana en segunda y tercera posición respectivamente.

Al cierre de octubre del 2019, el turismo internacional registró un decrecimiento del 7,9\% al arribar al país 3563005 visitantes, 303929 menos que en igual periodo del año pasado. 
En el mismo mes de octubre se recibieron 235613 visitantes, que representan un 27,8\% menos que en septiembre del 2018, debido principalmente al recrudecimiento del bloqueo por parte de Estados Unidos.

Durante la temporada alta los principales mercados europeos (Francia, Alemania, Reino Unido, Italia y España) registraron decrecimientos de entre 10\% y 13\% en la emisión de viajeros a Cuba, siendo una realidad que experimentan los destinos del Caribe, a decir de Michel Bernal Quicutis, director comercial del Ministerio de Turismo de Cuba (MINTUR), agregando que hay un reconocimiento entre los turoperadores de la mejora de la calidad del destino Cuba, con una infraestructura hotelera y de servicios competitiva con la del resto de los destinos del Caribe. (Cubadebate, 2019)

En los Lineamientos de la política económica y social del Partido y la Revolución, expuestos en el VII Congreso del Partido Comunista de Cuba en 2016, se definieron como parte de los factores con prioridad dentro de la estrategia cubana de desarrollo turístico, la diversificación de los mercados, productos y modalidades e incrementar el ingreso medio por turista, en aras de disminuir la dependencia de mercados específicos y ampliar la oferta turística. (Partido Comunista de Cuba, 2016) De esta forma, el estudio sobre la creación de nuevas modalidades capaces de diversificar la oferta, al tiempo que captan nuevos mercados y maximizan el gasto en el destino, se convierte en una necesidad imperiosa para el desarrollo turístico de la Isla.

Con la diversificación y el aumento de la competitividad entre los destinos, han surgido nuevas formas de hacer turismo, o como también se conocen modalidades turísticas. Y aunque el turismo y el comercio siempre han estado unidos y son dos actividades que tienen un impacto muy importante sobre el desarrollo de la sociedad, el surgimiento de la modalidad de turismo de compras ha generado grandes polémicas.

El turismo de compras se configura como una actividad que representa una parte muy importante de los ingresos. Ir de compras, como una actividad turística, ha aumentado significativamente en los últimos años (OMT, 2014). Numerosos estudios confirman que es una actividad en la que los turistas participan frecuentemente mientras viajan (JansenVerbeke, 1991), (Timothy \& Butler, 1995) (Yoon-Jung, Chia Kuen, \& Letho, 2004).

Las compras de los turistas siempre han existido; la práctica de adquirir bienes, recuerdos, moda, regalos, productos libres de impuestos, etc. en los viajes de esparcimiento ha sido habitual. Constituyen además actividades caracterizadas por el ambiente, la emoción, el placer y la oportunidad de interactuar con los vendedores locales (Tosun, 2007); por tanto, aunque las compras funcionan como una actividad de ocio, también suponen una oportunidad importante para exponerse a la cultura del anfitrión y ampliar la información sobre el destino turístico. Recientemente, el turismo de compras ha abierto nuevos cauces de desarrollo hasta convertirse en un motivo específico de desplazamiento a determinados destinos turísticos.

Elegir un destino turístico que te ofrezca la oportunidad de salir de compras funciona como una atracción adicional (Diamanche, 2003), (Moscardo, 2004). Distintos trabajos 
han reconocido que la actividad de compras funciona como una de las motivaciones más importantes de los viajeros (Reisinger \& Turner Lindsay, 2002), (Jansen-Verbeke, 1991), (Timothy \& Butler, 1995). Los turistas tienen motivos múltiples para realizar compras, incluyendo la diversión, la identidad, la satisfacción, el aprendizaje sobre las costumbres locales o el conocimiento de nuevas tendencias.

En cuanto a los mercados emisores de esta modalidad por concepto de gasto en compras libre de impuestos o tax free, China se ubica en el puesto número uno del ranking, con una cuota de mercado de 30,1\% en el 2018, según el sitio World Shopping Tourism Network (2019) experto en el tema.

"Diversas ciudades del mundo como Nueva York, Hong Kong, Londres y Dubái han convertido el comercio en una fuerte ventaja competitiva para el desarrollo de sus modelos turísticos, lo cual los ha posicionado como destinos urbanos primordiales y ha maximizado el ingreso medio por turista" (CBRE, 2016). De acuerdo a Hosteltur, (2017), la región de América Latina y el Caribe también ha apostado por el desarrollo de esta modalidad, en aras de diversificar la oferta y diferenciarse de la competencia, además de aumentar el ingreso turístico. A esta tendencia se han incorporado algunos destinos emergentes, quienes han diseñado estrategias y acciones encaminadas a su posicionamiento en la región.

En Cuba, se han comenzado a estudiar las premisas para la captación de esta modalidad, teniendo en cuenta el atractivo que representa y los factores claves de éxito para su puesta en marcha. Este inicio en el análisis ha posibilitado conocer los productos que más potencial tienen en la Isla para ser comercializados, que sean capaces de optimizar el ingreso al tiempo que se fortalece la imagen país.

"Los ingresos turísticos crecieron un 10,5\% frente al 16,2\% de crecimiento de los arribos, lo que refleja una disminución del ingreso por turista, que bajó de 1027 dólares en el 2005 a 722 en el 2017" (Rodríguez, 2018). Si vemos la evolución de este indicador, se puede observar una tendencia a la disminución, lo cual es contrario a los objetivos prioritarios del sector turístico cubano. De esa forma, es necesario crear ofertas extrahoteleras y modalidades complementarias más eficientes.

Sin embargo, el desarrollo de una nueva modalidad en un destino es una labor compleja, debido a la necesidad de crear infraestructuras, captar nuevos segmentos, posicionarse en el mercado, entre otros, que en el caso de Cuba se agrava por la presencia del bloqueo económico-comercial del gobierno de Estados Unidos, sumado al actual retroceso en las relaciones bilaterales entre ambos países.

Actualmente se conocen o se investigan los elementos que desde el punto de vista interno son claves para el desarrollo del turismo de compras en La Habana, lo cual ha hecho necesario el análisis de los factores críticos de éxito, el diagnóstico del comercio minorista y mayorista, así como la logística y las cadenas de suministro. Sin embargo, la perspectiva de los competidores potenciales en lo referente a las mejores prácticas que lideran la actividad, y las brechas existentes, el análisis de la atractividad del mercado, la competitividad del destino, y las potencialidades y limitantes reales del mismo para el 
desarrollo de la modalidad, no han sido estudiadas con el fin de identificar los elementos que se necesitan poner en marcha desde el punto de vista de la oferta y la demanda.

Teniendo en cuenta lo anteriormente planteado es que dicha investigación tiene como objetivo general: Determinar estrategias para el desarrollo de la modalidad de turismo de compras en el destino la habana.

\section{Importancia de los resultados:}

Con la realización de esta investigación se pretende cumplir con los Lineamientos de los 6to y 7 mo Congresos del Partido y la Revolución referidos a la diversificación de los servicios complementarios al alojamiento turístico y a la captación directa de divisas. De igual forma con esta investigación se busca contribuir a la diversificación de la oferta turística de La Habana, eliminar la estacionalidad y aumentar los ingresos de divisas al país. De acuerdo a lo expuesto anteriormente, Turismo y comercio son dos actividades que tienen un impacto muy importante sobre el desarrollo de la sociedad, cuando los turistas viajan para comprar tienen el placer y la oportunidad de interactuar con los vendedores locales favoreciendo el intercambio cultural, estas actividades no solo benefician a los visitantes sino a los anfitriones, pues genera más empleos en la región donde se desarrolla. En Cuba tanto el sector privado como el estatal pueden resultar beneficiarios, aprovechando la riqueza cultural de la mayor de las Antillas.

\section{Acápite 1. Turismo, modalidades turísticas y turismo de compras.}

\section{El turismo. Modalidades turísticas.}

Uno de los mayores desafíos de la investigación en el turismo ha sido la definición del término en sí, debido a los diferentes enfoques que los autores le han dado al mismo. Estos mencionados enfoques vienen dados principalmente por la naturaleza multidisciplinaria del turismo y la presencia de elementos económicos, psicosociológicos, histórico-culturales y geográfico-espaciales, que el Dr. Ramón Martín ha identificado como los grandes componentes del turismo. (Martín, 2009)

Según la Organización Mundial del Turismo, en 1994, "el turismo comprende las actividades que realizan las personas durante sus viajes y estancias en lugares distintos a su entorno habitual, por un periodo de tiempo consecutivo inferior a un año, con fines de ocio, negocios y otros". (Citado por Vento, 2019).

Según Martín (2009) el turismo se define como el conjunto de fenómenos y relaciones económicas, psico-sociológicas y medioambientales que se generan entre las entidades vinculadas a los viajes desde el lugar emisor, las entidades proveedoras de servicios y productos en el lugar de destino, los gobiernos de los lugares emisores-receptores y las comunidades locales de acogida, con motivo del viaje y estancia de visitantes temporales en un destino diferente a su lugar de residencia habitual.

Por razones prácticas, los especialistas en turismo han establecido una clasificación del turismo en modalidades, para facilitar su comprensión, lo que permite saber las motivaciones de viaje de cada turista y brindarles un servicio más personalizado de acuerdo a sus necesidades. 
La modalidad turística se puede definir como una forma específica de turismo con relación a otra. Es decir, una modalidad turística está referida al aspecto singular o modo que motiva la práctica del turismo. Esto se traduce en que no todos los turistas practican la misma forma de visitar lugares ajenos a sus sitios de residencia, ni pernoctan en un mismo tipo de alojamiento, ni están motivados a viajar por una misma causa. Por ello se hace una clasificación del turismo en función de los modos como se practica o de las causan que lo motivan. (Manapocatl, 2016)

Llanes (2018) define las modalidades turísticas como "el compendio de motivaciones, actividades, atractivos, destinos y segmentos de la demanda, con una correlación directa y análoga que facilita su comercialización".

De acuerdo a Martín (2009) las modalidades turísticas se definen a partir de la clasificación de dichas actividades, siendo denominadas además como productos turísticos o nichos turísticos. Aunque también es usual clasificarlas a partir del criterio de turismo convencional o tradicional (masivo casi siempre vinculado al turismo de sol y playa) y turismo alternativo o diferenciado.

Las modalidades turísticas son el resultado de una estrecha relación entre motivaciones y actividades realizadas en destinos específicos con atractivos y recursos específicos, que definen una determinada forma de hacer turismo y facilitan su posterior comercialización. (Fernández-Criado, 2019)

El Shopping o turismo de compras es una modalidad en desarrollo y supone un gran incentivo para el destino. Nace a partir de la costumbre de comprar cuando el turista está en pleno viaje. Para quienes practican esta modalidad no supone lo mismo comprar un producto en su ciudad de residencia habitual que en el lugar de procedencia original de dicho producto, sumado esto al significativo aporte de las grandes marcas para muchos viajeros. Es un tipo de turismo que puede complementarse con otros, como la modalidad cultural, y es también un gancho para coleccionistas.

\section{Turismo de compras.}

Moscardo (2004) sugirió que todo el consumo que los turistas realizaran en servicios específicos era turismo de compras, mientras que Timothy (2004) dice que el consumo no se trata solo de productos, es sobre lugares, espacios y tiempos. Este último plantea que, de la relación entre el comercio y el turismo, se desprenden dos actividades fundamentales que constituyen las caras de una misma moneda: el turismo de compras y las compras de turismo. El propio autor explica que la diferencia radica en que el primero se refiere al viaje motivado

por las compras, mientras que la segunda se refiere a la adquisición secundaria e incidental por parte de los turistas de productos en un destino. 
El turismo de compras permite a los visitantes llevar a casa la evidencia física, los recordatorios tangibles de una experiencia que difiere a su rutina diaria. (Gordon, 1986) Desde la perspectiva del turista, los bienes adquiridos son una prueba de las experiencias vividas. Las compras son a menudo un consumo simbólico en el que los productos adquieren un significado especial. (Graburn, 1980) (Littrell, y otros, 1994)

Sobre la base de estas definiciones es que se establece el concepto de la Organización Mundial del Turismo (2014). Dicha definición plantea que el turismo de compras es "una forma contemporánea de turismo realizado por individuos para los que la adquisición de bienes fuera de su lugar de residencia es un factor determinante (aunque no necesariamente único) en su decisión de viajar”. (OMT, 2014)

Magadán \& Rivas (2014) exponen que la motivación del turismo de compras no es únicamente el conseguir productos exclusivos o únicos, que no se encuentren en el lugar de origen o cuyo precio sea mucho más bajo, sino que se trata, en parte, del valor simbólico que estos bienes representan.

En el año 2015, un informe presentado por Ernst \& Young S.L. explica que esta modalidad turística está principalmente vinculada a destinos urbanos, aunque no de manera exclusiva, la cual permite al turista acceder a productos bajo condiciones que no encuentra en su lugar de residencia habitual, ya sea por disponibilidad o exclusividad de la oferta o por un mejor precio. A esto agregan que cada vez existen más turistas que viajan para comprar, y que el turismo de compras se ha convertido en pocos años en el producto turístico de mayor crecimiento del sector, y el que más expectación está suscitando a escala internacional debido a su gran potencial. Por ello, resumieron los beneficios de la modalidad de la manera siguiente:

- Capacidad de fidelización de los turistas: lograr una oferta atractiva de compras, permite que los clientes seguidores de las grandes marcas, o de los productos locales, vean en el destino una fuente segura para la satisfacción de sus necesidades, lo que los hace repitentes. A medida que los destinos consoliden sus ventajas competitivas en este segmento irán consiguiendo la fidelización de sus clientes.

- $\quad$ Elevación del gasto turístico: siendo la adquisición de productos el objetivo fundamental que persigue esta modalidad, el gasto se coloca en el centro de la motivación de este tipo de turistas. Así, si la propuesta es buena, los clientes no se medirán en adquirir lo que vinieron buscando, además de las compras por impulso. Sumado a ello, este segmento gasta de 3 a 4 veces más que los turistas convencionales (Choi, 2016) y permanece más tiempo en el destino (Jansen-Verbeke, 1991), elevando así el consumo turístico.

- Diversificación de la oferta del destino: mediante esta modalidad se pueden desarrollar nuevos productos para nuevos mercados. Además, sirve para consolidar la imagen de ciudades y generar un posicionamiento positivo de las mismas.

- Extensión del impacto del turismo a otros sectores económicos: el carácter multisectorial del turismo se acentúa más aún con esta modalidad, donde se dinamizan productores tales como artesanos, agricultores, diseñadores, 
artistas y empresas electrónicas, además de proveedores y minoristas, en fin, todos los integrantes de la cadena de suministros.

- $\quad$ Efecto desestacionalizador: esta modalidad no está asociada al clima ni a los recursos naturales, por lo que puede ser potenciada en los meses donde ocurren los mayores descensos de los flujos turísticos del destino.

- Generación de ingresos en el territorio: el desarrollo de esta actividad, principalmente la que potencia los productos locales, es una fuente de empleo para los miembros de la comunidad, además que constituye un canal ideal para la venta de muchos de los productos que en dicha comunidad se crean. Por otra parte, mediante los impuestos que el Estado carga, también se favorece el desarrollo económico del territorio.

Sobre estos beneficios, García (2016) agrega que es una actividad que hace al sector comercial local más competitivo, ayuda a consolidar la imagen de los destinos y a generar un posicionamiento positivo y puede ser fundamental para la rehabilitación o reposicionamiento de zonas urbanas deprimidas.

Según Abu El Enain (2017) los turistas están seleccionando de forma gradual a las compras como un método para practicar actividades típicas de la cultura del destino, implicándose con los productos domésticos y los artesanos nativos. De esta forma se manifiesta la relación entre esta modalidad y el turismo cultural (en sus diversas aristas).

La autora concuerda con las fuentes anteriormente citadas, estableciendo que siempre que se hable de turismo de compras se hace referencia a una modalidad turística contemporánea realizada por individuos cuya principal motivación de viaje (puede que no la única) es la compra de productos exclusivos o únicos, que no se encuentran en su lugar de residencia habitual o el precio en que se ofertan es sensiblemente más bajo, con un alto valor simbólico. Se desarrolla en destinos urbanos principalmente, los que no necesariamente tienen que ser un paraíso de compras.

Las principales tendencias de la modalidad según la Organización Mundial del Turismo, (2014); Ernst \& Young, (2015); y CBRE, (2016) se relacionan a continuación:

- Los países asiáticos liderarán el crecimiento del turismo emisor tanto en gasto como en flujos, con China a la cabeza.

- $\quad$ El crecimiento de esta modalidad será muy superior al del resto debido a que las compras son la motivación principal, o una de las principales, de los mercados emergentes que liderarán el crecimiento del turismo internacional.

- Estados Unidos tendrá un papel significativo dentro de las emisiones turísticas, pero también en la captación de este segmento de demanda.

- Se posicionarán mercados emergentes, no solo asiáticos, sino también latinoamericanos con propuestas innovadoras y experiencias de compras gratificantes.

En cuanto al análisis de los principales países emisores de turismo de compras, se toma como referencia al sitio web World Shopping Tourism Network (2019) el cual cita a Global Blue (que es la operadora internacional líder de tax free), revelando que, de 
acuerdo con el total de las devoluciones de impuestos gestionadas por la misma, los mayores compradores internacionales son los turistas procedentes China, Rusia Y estados Unidos.

El estudio elaborado por The Economist Intelligence Unit y Global Blue (2012) clasifica a los mercados emisores en función de dos criterios: número de transacciones y el importe de las mismas. Según estos criterios:

- $\quad$ China y Rusia realizan el mayor número de transacciones.

- $\quad$ Tailandia, Hong Kong e Indonesia son los que realizan compras de mayor importe, junto con China.

- Japón, Estados Unidos y Malasia realizan también un número importante de transacciones, pero por importes inferiores a los chinos, tailandeses o indonesios.

En la bibliografía consultada, los autores habitualmente coinciden en los principales destinos turísticos de compras en el mundo en sus diferentes listas, sin embargo, los mismos difieren en el orden, pues para elaborar estos rankings o tops cada uno se vale de sus propias experiencias de compras o los comentarios y referencias de personas que han visitado esos lugares con estos propósitos.

Así, para determinar los principales destinos turísticos de compras se analizaron los estudios realizados por Global Blue (2012), Kim (2013), J.R. (2015), Shironosov (S/A) y Madrid (2017). De esta manera, se puede inferir entonces que los principales destinos turísticos de compras a nivel mundial sin tener en cuenta el orden son: Londres, París, Barcelona, Madrid, Berlín, Milán, New York, São Paulo, Buenos Aires, Kuala Lumpur, Shanghái, Hong Kong, Beijing, Dubái y Tokio. (Chávez, 2019)

Según las definiciones anteriores aportadas por los estudiosos de este tema, se pudo apreciar que a pesar de que dichos autores caractericen esta modalidad turística desde puntos de vista diferentes, todos coinciden en que el Turismo de Compras no debe solo contemplarse como el producto a adquirir por el turista, es fundamental que los destinos resulten atractivos y de fácil acceso para este segmento de la demanda turística, y que además existen una serie de factores que posibilitan la elección de un destino de compras: la exclusividad de la oferta comercial, el precio de la oferta comercial, la conectividad del área internacional, la seguridad del destino, tasa de devolución de impuestos, la calidad y limpieza del entorno, la oferta de alojamiento, la oferta de restauración y la oferta cultural y de ocio.

\section{Acápite 2: El comercio minorista en La Habana. Estrategia Metodológica.}

El comercio de productos en Cuba, está rectorado por el Ministerio de Comercio Interior (MINCIN), Organismo de la Administración Central del Estado (OACE) encargado de la aplicación de la Política del Estado y el Gobierno en cuanto al Comercio Interno de las actividades comerciales que operan en moneda nacional y en moneda libremente convertible, en los sectores estatal, cooperativo, mixto y privado (Gobierno de la 
República de Cuba, S/A). El objetivo central del MINCIN es la organización de la circulación, el almacenaje y el intercambio de mercancías y servicios en el territorio nacional. Para la realización de estas funciones, el MINCIN cuenta en todo el país con una amplia red de almacenes de Comercio Mayorista para la compra, almacenaje y venta de mercancías, de productos nacionales y de importación, con destino a las entidades que ejercen el Comercio Minorista y la Gastronomía; tiendas de Comercio Minorista, para la venta de mercancías, de productos nacionales o de importación, con destino a la población, para su uso y consumo; unidades de Gastronomía, para la prestación de los servicios gastronómicos que se brindan en establecimientos comerciales destinados a estos fines (restaurantes, cafeterías, centros nocturnos, bares, entre otros); y unidades de Servicios Comerciales destinadas a la satisfacción de necesidades de carácter social, individual o colectivo (Gobierno de la República de Cuba, S/A).

Sin embargo, esta OACE no es la única que participa en la actividad comercial de la Isla, coexistiendo junto a otros organismos y entidades independientes, lo que complica la regulación y el control de la actividad. De esa forma existen organismos como el Ministerio de Cultura (MINCULT), el Ministerio de Turismo (MINTUR), el Ministerio de las Fuerzas Armadas Revolucionarias (MINFAR) y el Ministerio del Interior (MININT), a los cuales se subordinan entidades comercializadoras de productos al detalle y de forma mayorista.

En cuanto a la estructura del comercio de bienes en Cuba, según García (2012), en primer lugar, se encuentra el comercio estatal cuyos recursos materiales y financieros pertenecen al Estado. Este tipo de comercio desempeña el papel rector y dominante en el sistema cubano. Este a su vez está compuesto por cinco grandes grupos de instituciones: las empresas de comercio minorista normado, que aseguran la venta de la canasta básica a precios especiales y por igual a todos los ciudadanos; las empresas de comercio minorista, que se encargan de la comercialización de productos liberados, en moneda nacional, tanto industriales, como agropecuarios, a precios superiores a los normados; las actividades de comercio minorista de organismos estatales y entidades, resultado de autoconsumo, estímulos, mercados para sectores específicos y otros; las empresas de comercio minorista que venden sus mercancías en divisa y en moneda nacional; y el sistema de comercio mayorista y de importación.

En segundo lugar se encuentra el comercio no estatal que contiene el comercio llamado Mercado Agropecuario, que comprende aquellos productos agropecuarios que comercializan las cooperativas y los campesinos privados como resultado del excedente de producción que poseen después de cumplir sus obligaciones con el Estado; el comercio privado, en el cual un sector de la población (cuentapropistas), participa en la comercialización de productos elaborados o no por ellos mismos; y el comercio sumergido, ilegal pero que existe (García, 2012).

En los últimos años se ha observado un aumento en el papel del comercio privado dentro de la estructura del comercio minorista en la Isla, amparado por la apertura del trabajo 
por cuenta propia y la intención expresa del Estado para el desarrollo de las formas de gestión no estatal en coexistencia con las estatales. Como parte de los Lineamientos de la política económica y social aprobados en el VII Congreso del Partido Comunista de Cuba (PCC), en el lineamiento 252, perteneciente a las políticas para el comercio, se expresa se expresa que es necesario: "Continuar la introducción gradual, donde se considere necesario, de formas no estatales de gestión en el comercio" (Partido Comunista de Cuba, 2016). Sin embargo, no se han creado todas las condiciones para el desarrollo de este nuevo sector, principalmente en su aseguramiento logístico. Al respecto los lineamientos 248 y 249 abordan la temática de la gestión mayorista y el aprovisionamiento de las formas de gestión no estatal.

En el semanario económico Opciones (2017), Orlando Nicolás Hernández Guillén, presidente de la Cámara de Comercio de la República de Cuba, manifestó que el sector comercial presentó avances en relación con el año precedente, a pesar de la realidad que impone el bloqueo económico, comercial y financiero de los Estados Unidos ahora recrudecido, además de otras circunstancias de carácter subjetivo como las trabas burocráticas que demoran los procesos de factibilidad y los prejuicios respecto a la inversión extranjera asociada con un retorno al capitalismo.

Otro aspecto que es necesario destacar es el de la dualidad monetaria, ya que en la Isla coexisten dos monedas el CUC y el CUP, cuyo valor está en la razón 1:24. Si bien antiguamente el CUC era la moneda que se usaba para la adquisición de bienes en las tiendas de la red de divisas, actualmente estas operaciones también se pueden realizar en CUP o moneda nacional, ya que el gobierno está trabajando por la unificación monetaria. En cuanto a los métodos de pago, aunque el efectivo continúa siendo la principal forma, se han empezado a desarrollar métodos como el pago mediante tarjetas magnéticas en algunos establecimientos de la red de tiendas de moneda libremente convertible, aunque esto excluye a las tarjetas emitidas y gestionadas por bancos estadounidenses, debido al bloqueo económico y financiero antes mencionado (Cifre, 2017).

\section{Metodología}

\section{Estrategia Metodológica}

Para la puesta en marcha de la investigación fue necesario establecer un enfoque metodológico que permitiera de manera ordenada y lógica, cumplir el objetivo trazado al inicio.

\section{Tipología de la investigación y fuentes de información}

De acuerdo con Perelló, (2005), en su clasificación de las investigaciones científicas por formas y tipos, la presente investigación es aplicada, ya que depende de los descubrimientos y aportes teóricos de las investigaciones puras, buscando afrontar la teoría con la realidad y la práctica. Además, posee un enfoque cuantitativo y cualitativo ya que persigue hacer una evaluación de los destinos comparados desde un punto de vista 
de la cantidad de determinados factores, pero también de la calidad de otros factores, apoyándose para ello en indicadores cuantitativos y cualitativos, dando lugar a una mejor comprensión del desempeño de los destinos de referencia; así como la aplicación de la Matriz Atractividad- Competitividad-Modalidades Turísticas.

En cuanto a las fuentes de información las mismas pueden clasificarse en primarias y secundarias según su forma de obtención. De acuerdo a Montalvo y otros, (2005), la información primaria es aquella que no está elaborada ni recogida por lo que es necesario llevar a cabo su captura, de ahí que se obtenga mediante el contacto directo con el objeto de estudio. Por su parte las fuentes secundarias, son aquellas que recogen información ya procesada y elaborada previamente, por lo que se obtienen mediante el contacto indirecto con el objeto de estudio. Para el desarrollo del presente trabajo, las fuentes utilizadas sujetas a la anterior clasificación son las siguientes:

Fuentes primarias: Entrevistas a especialistas y guías turísticos, encuestas a expertos y turistas, y observación no participativa.

Fuentes secundarias: Comentarios de viajeros en Trip Advisor, sitios en Internet, trabajos de investigación referentes al tema de las compras y el comercio, libros, revistas y publicaciones oficiales en la web.

Estrategia metodológica de la investigación

El presente trabajo siguió una estrategia metodológica que consta de cuatro etapas y sus diferentes fases, donde se aplicaron diferentes métodos teóricos y empíricos (Tabla 1).

Tabla 1: Estrategia metodológica de la investigación.

\begin{tabular}{|c|c|c|}
\hline ETAPAS & FASES & MÉTODOS Y \\
\hline \multirow[t]{2}{*}{ I. Estudio del turismo de compras } & $\begin{array}{l}\text { 1. Definición de la } \\
\text { modalidad. }\end{array}$ & $\begin{array}{l}\text { - Análisis } \\
\text { documental y }\end{array}$ \\
\hline & $\begin{array}{l}\text { 2. Comportamiento a } \\
\text { nivel internacional }\end{array}$ & $\begin{array}{l}\text { - Análisis y } \\
\text { síntesis } \\
\text { - Análisis } \\
\text { Inductivo- } \\
\text { deductivo }\end{array}$ \\
\hline \multirow{3}{*}{$\begin{array}{l}\text { II. Desarrollo de la herramienta de } \\
\text { benchmarking. Destino objeto de } \\
\text { estudio y destinos de referencia de } \\
\text { América Latina. }\end{array}$} & 1. Fase de planificación. & $\begin{array}{l}\text { - Análisis } \\
\text { documental } \\
\text { - Búsqueda }\end{array}$ \\
\hline & 2. Fase de análisis & $\begin{array}{l}\text { referencial en } \\
\text { Internet }\end{array}$ \\
\hline & 3. Fase de acción & $\begin{array}{l}\text { - Análisis } \\
\text { comparativo } \\
\text { - Entrevista } \\
\text { - Observación } \\
\text { no } \\
\text { participativa }\end{array}$ \\
\hline \multirow{3}{*}{$\begin{array}{l}\text { III. Aplicación de la Matriz A-C-M } \\
\text { para los mercados rusos y chinos }\end{array}$} & 1. Definir atributos por & - Análisis \\
\hline & 2. Representación gráfica & - Encuesta \\
\hline & 3. Retos y oportunidades & -Entrevista \\
\hline
\end{tabular}




- Método o
juicio de
expertos
- Métodos
matemáticos-
estadísticos
egias.

Fuente: Elaboración propia.

\section{Acápite 3: Resultados del Benchmarking y la Matriz A-C-M. Propuesta de estrategias.}

Etapa II. Desarrollo de la herramienta de benchmarking. Destino objeto de estudio y destinos de referencia de América Latina.

\section{Fase de planificación}

- Caracterización del panorama comercial de La Habana.

En cuanto a los tipos de establecimientos minoristas presentes en el destino La Habana, se pueden encontrar fundamentalmente los centros comerciales (Galerías Paseo, Plaza de Carlos III, 5ta y 42); los supermercados (3ra y 70), las tiendas de autoservicio, las tiendas especializadas y las boutiques. Sin embargo, no existen en el destino los hipermercados, los grandes almacenes, las tiendas de descuento, los outlets, ni las tiendas de conveniencia (Cifre, 2017). Según el Ministerio de Comercio Interior, (2015), los principales minoristas del destino son Caracol S.A., TRD Caribe, Tiendas Habaguanex, Tiendas Panamericanas y Coral Negro del grupo CIMEX, las Tiendas del Grupo PALCO, Tiendas Artex, así como las organizaciones Asociación Cubana de Artesanos Artistas (ACAA) y el Fondo Cubano de Bienes Culturales (FCBC), que representan el quehacer artístico de un gran número de creadores cubanos.

A continuación, se exponen algunos elementos que han permitido relacionar las categorías de comercio y turismo, o han sido atractivos en este sentido, de acuerdo a Cifre, (2017) y Opciones, (2015):

- Creación de una cadena de tiendas, Caracol S.A., encargada de la comercialización de mercancías fundamentalmente para el turismo.

- Presencia de productos genuinos de alto valor comercial como el tabaco, el ron, el café, la guayabera, la artesanía, las artes plásticas y las antigüedades.

- Apertura del sector cuentapropista y el desarrollo de espacios comerciales de alto valor turístico, como las llamadas ferias (La Rampa, Almacenes de San José, Artesanos de Obispo, entre otros).

- Desarrollo de eventos que promueven y/o venden productos cubanos tales como la Feria Internacional de Artesanía (FIART), el Festival del Habano, la Feria del Libro y la Semana de la Moda en La Habana.

- Creación de la marca Casa del Habano, para la comercialización de este producto significativo. 
- La creación de tiendas de marcas internacionales en los principales hoteles del destino (Comodoro, Gran Manzana, Sevilla).

- Creación de una tienda Samsung en el destino en unión con la cadena de tiendas cubana TRD.

- La no existencia del Impuesto sobre el Valor Añadido (IVA) a los productos que se comercializan en la Isla.

- El desfile de la casa de modas Chanel en La Habana.

- El desarrollo de nuevas marcas de diseño textil de gran aceptación, como Clandestina y Puntozero.

- El aumento en la llegada de cruceristas al destino, que representan un mercado potencial para las compras.

- Selección y caracterización de los destinos de referencia

Luego de una revisión exhaustiva en Internet que incluyó el análisis de posicionamiento en el buscador Google, de comentarios de clientes en Trip Advisor, de foros y blogs sobre las compras en América Latina, de informes de la OMT sobre los destinos de turismo de compras e informes de Global Blue y Hosteltur, se identificaron los principales destinos donde se practica la modalidad en la región, siendo un total de 11 los destinos más atractivos y exitosos. Debido a los recursos y el tiempo con que se cuenta para la realización de esta investigación, solo se tomó una muestra de 5 destinos, utilizando un muestreo no aleatorio por conveniencia. Para la selección de la muestra se tuvieron en cuenta los siguientes criterios:

- Paisaje fundamentalmente urbano, con amplio desarrollo del turismo de ciudad.

- Posicionamiento como destinos de compras en todos los sitios consultados.

- Disposición de información en sitios oficiales de Internet sobre los destinos escogidos.

- Eliminación de los destinos donde el turismo de compras se realiza fundamentalmente en las fronteras con otros países, debido a que La Habana no tiene fronteras internacionales.

A partir de lo antes expuesto, los 5 destinos seleccionados para la realización del benchmarking fueron las ciudades de Sao Paulo, Santiago de Chile, Ciudad Panamá, Buenos Aires y Ciudad de México

- Indicadores de benchmarking

Global Blue, (2012) llevó a cabo una propuesta de indicadores cuantitativos, cualitativos y mixtos, llamados The Globe Shopper Index para valorar el potencial de un destino con relación al turismo de compras. Para la presente investigación se tomaron como base dichos indicadores y se adaptaron a partir de los factores claves identificados en Timothy, (2005); Organización Mundial del Turismo, (2014); Ernst \& Young, (2015) y Cifre, 
(2017) teniendo en cuenta el alcance, las posibilidades y los objetivos del presente trabajo. De esa forma se obtuvieron 17 indicadores que quedaron agrupados en 4 categorías generales: sector comercial, asequibilidad, conveniencia e infraestructura y ocio.

\section{Fase de análisis}

Como se puede apreciar en las figuras 1 y 2, el destino Ciudad de México lidera de forma global, los indicadores analizados para evaluar el atractivo del turismo de compras, alcanzando un promedio de 81.71. La ciudad posee grandes atractivos para llevar a cabo esta modalidad y convertirse en un destino de referencia global. La ciudad de Buenos Aires (65.43), se ubica segunda en esta comparación, lo que explica la fama internacional que posee, sin embargo, vale aclarar que el resultado

global no explica algunos elementos cruciales que han disminuido el atractivo de la ciudad y que se pueden apreciar mejor en la categoría sector comercial y los indicadores precio de los bienes y porciento de IVA. Sao Paulo (53.58), ocupa el tercer lugar en este estudio, alcanzando un resultado por encima de la media; además se puede observar en la figura 1 que es la segunda ciudad en la categoría sector comercial, lo cual explica el auge de la modalidad y el posicionamiento de la misma a escala global. En el caso de Santiago de Chile (44.53) y Ciudad Panamá (35.79), se muestra cómo son destinos emergentes, que están creando políticas para el desarrollo de la modalidad y la atracción de flujos de turistas de compras.

En el caso de La Habana (20.18), en la figura 1 se puede apreciar el papel destacado en la categoría conveniencia. Sin embargo, existen brechas importantes en las categorías sector comercial, infraestructura y ocio y asequibilidad, cuya disminución es prioritaria para el desarrollo de la modalidad en el destino.
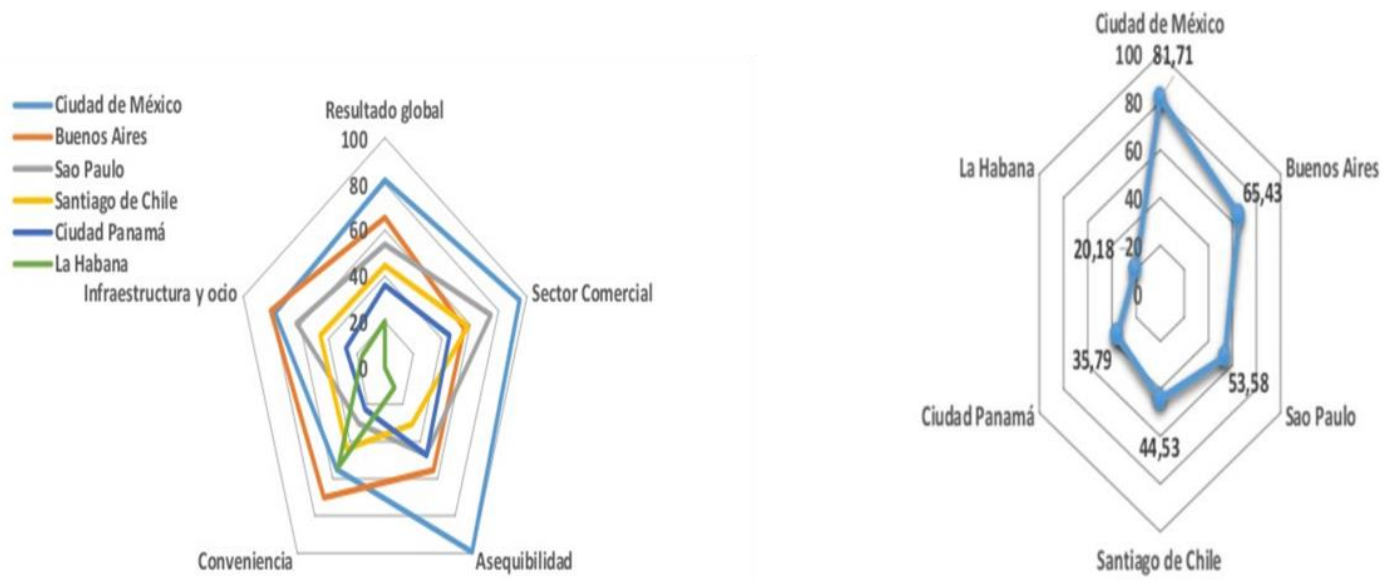

Figura 1. Resultado global por categorías. Figura 2. Resultado global del benchmarking

Fase de acción 


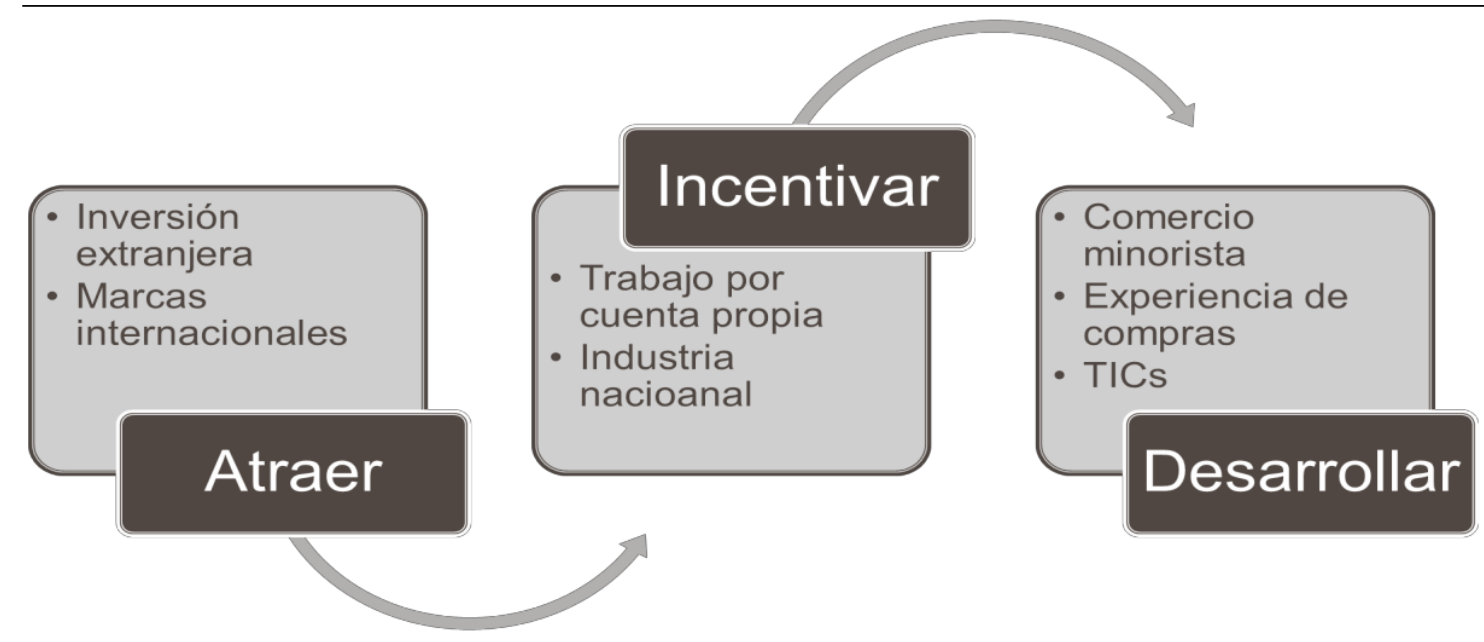

Figura 3. Retos y oportunidades a partir del Benchmarking.

Fuente: Elaboración propia.

Etapa III. Aplicación de la Matriz A-C-M para los mercados rusos y chinos.

Fase 1. Definir atributos por variables.

\section{Atractividad}

Tabla 2. Análisis de la atractividad de los mercados.

\begin{tabular}{lll}
\hline & $\begin{array}{l}\text { Mercado } \\
\text { Ruso }\end{array}$ & Mercado Chino \\
\hline Cluster 1 & $\begin{array}{l}149 \text { casos: } \\
\text { Hombres de } \\
\text { compras }\end{array}$ & $\begin{array}{l}\text { Caribe. } \\
\text { Con casos: Nuevos descubridores del }\end{array}$ \\
& $\begin{array}{l}132 \text { casos: } \\
\text { Vacacionistas } \\
\text { repitente }\end{array}$ & 120 casos: Chicas a la moda \\
& $\begin{array}{l}91 \text { casos: } \\
\text { Chicas a la } \\
\text { moda }\end{array}$ & 118 casos: Amigos de Cuba \\
Cluster 3 & $\begin{array}{l}\text { Cluster 1 } \\
\text { Cluster más atractivo }\end{array}$ & Cluster 2 y 3 \\
$\begin{array}{l}\text { atendiendo a rentabilidad } \\
\text { económica y accesibilidad } \\
\text { del segmento }\end{array}$ & & \\
$\begin{array}{l}\text { Atractivo estructural del segmento a partir del análisis de las Cinco Fuerzas de la } \\
\text { Competitividad de Michael Porter }\end{array}$ & \\
\hline
\end{tabular}

Fuente: Elaboración propia. 
El análisis de la competitividad del destino La Habana se realizó a partir del segundo eje de la matriz de atractividad - competitividad - modalidad turística. Para ello se analizaron cada uno de los elementos contenidos en el Diamante de la competitividad de Porter.

1. Condiciones de los factores de explotación turística

- Atractivos y Recursos Turísticos

- Infraestructura y servicios básicos

- Infraestructura de alojamiento

- Servicios extrahoteleros (restauración, ocio y recreación, comercio minorista, agencias de viajes y turoperadores, información turística, actividad de apoyo al turismo, tecnología y recursos humanos)

2. Condiciones de funcionamiento de las empresas del sector

3. Condiciones de la demanda captada por el destino.

4. Condiciones de los sectores conexos y de apoyo (transporte, servicios médicos, comunicaciones, abastecimiento de agua y alcantarillado y saneamiento)

\section{Modalidad turística: Turismo de compras.}

Con el fin de analizar la modalidad turística de Turismo de Compras se utilizó el tercer eje de la matriz de atractividad - competitividad - modalidad turística y se tuvo en cuenta cada uno de los elementos contenidos en dicha matriz.

1. Recursos y atractivos turísticos exclusivos para la modalidad.

2. Requerimiento de inversión.

3. Existencia de sistemas de capacitación y formación del personal en su totalidad para la modalidad

4. Homogeneidad intracluster y Heterogeneidad intercluster

5. Marco regulatorio y presiones externas

\section{Fase 2. Representación gráfica.}

Una vez analizadas cada una de las variables se prosiguió a graficarlas con el fin de aplicar la matirz de atractividad - competitividad - modalidad turística. Por lo que, al representarlas en la matriz de tres ejes quedó de la siguiente manera. 


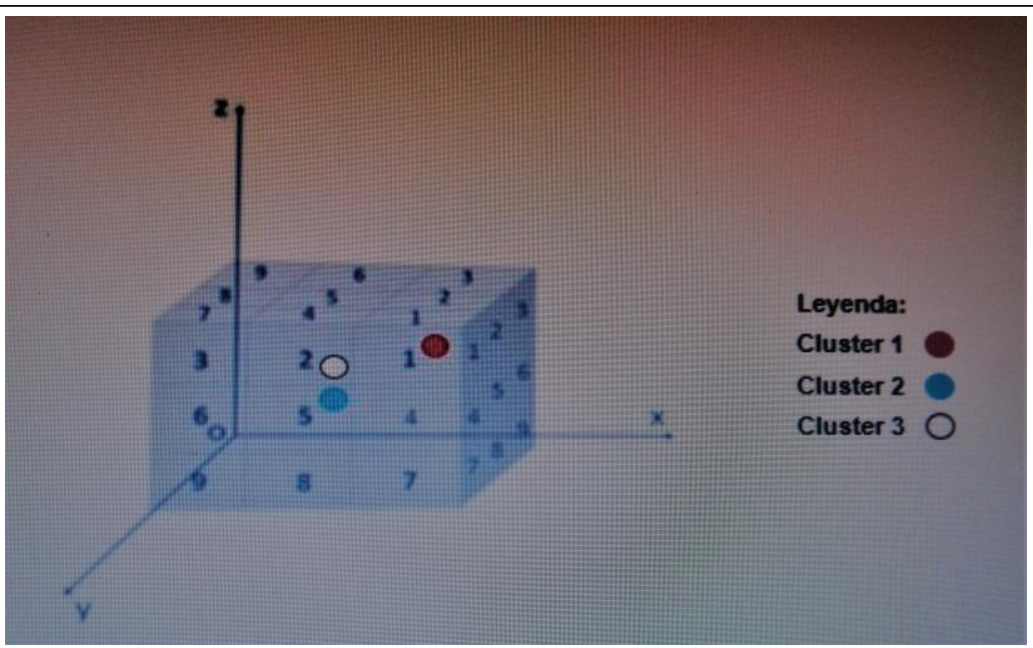

Figura 4. Mercado ruso: clúster no.1 "Hombres de compras".

Fuente: Elaboración propia.

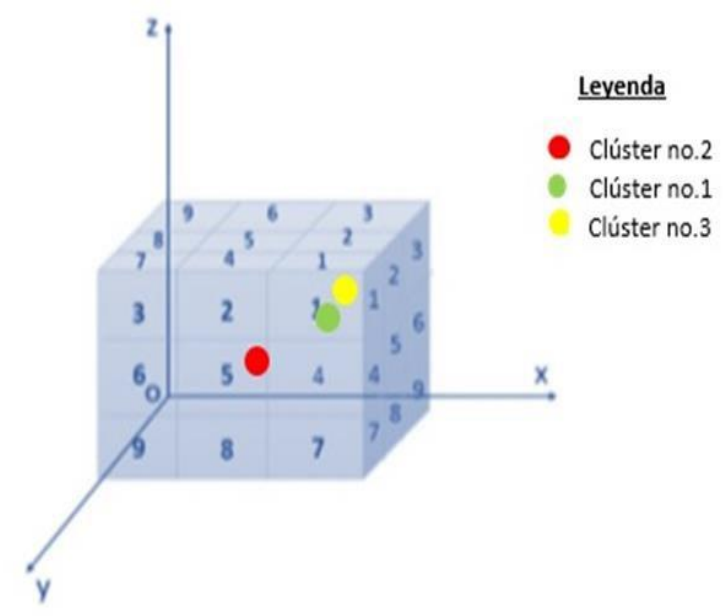

Figura 5. Mercado chino: clúster no.3 "Amigos de Cuba".

Fuente: Elaboración propia

Fase 3. Retos y oportunidades.

Tabla 3. Oportunidades y retos a partir de la Matriz A-C-M.

\begin{tabular}{ll}
\hline \multicolumn{1}{c}{ Oportunidades } & \multicolumn{1}{c}{ Retos } \\
\hline Productos motivadores de viajes & Desarrollar el \\
Espacios comerciales para el desarrollo del turismo & Revitalizar la \\
Nuevos mercados y segmentos de demanda & Proyeccio n \\
Exclusividad dentro de su oferta comercial, cultural y & Desarrollar \\
\hline
\end{tabular}

Fuente: Elaboración propia.

Etapa IV. Propuesta de estrategias. 


\section{Estrategias para el Turismo de Compras}

- Diseño de productos turísticos de compras, acordes a las exigencias y características de los segmentos de demanda, y que combinen el sector comercial estatal y el no estatal, que resalten las producciones auténticamente cubanas.

- Creación de paquetes turísticos que combinen varias ciudades del país, alargando así la estancia de los turistas y expandiendo el impacto de la modalidad a distintas regiones.

- Comercialización del producto de Turismo de Compras en los destinos emisores.

- Desarrollo de alianzas estratégicas que permitan la revitalización del sector productivo y comercial del destino.

\section{Conclusiones}

- El Turismo de Compras, es una modalidad que evoluciona cada vez más, y que se ha convertido en una vía potencial para el desarrollo de algunos destinos turísticos.

- Existe un gran número de retos para el desarrollo de la modalidad de turismo de compras en La Habana, sin embargo, existen oportunidades que deben ser aprovechadas en este sentido.

- La materialización de las estrategias definidas, permitirá establecer la modalidad de Turismo de Compras en La Habana.

\section{Referencias bibliográficas}

Abu El Enain, E. y Yahia, S. 2017. Benchmarking the Egyptian shopping tourism sector against international best practices in Dubai, UAE. El Cairo : European Journal of Multidisciplinary Studies, 2017.

Blázquez, J., Esteban, Á. y Molina, A. 2011. Benchmarking de destinos turísticos: diferencias de calidad del servicio según el producto turístico comercializado. Castilla-La Mancha : Papeles de Economía Española, 2011.

Camp, R. 1989. Benchmarking: The search for industry best practices that lead to superior performance. Wisconsin : ASQC Quality Press, 1989.

CBRE. 2017. Turismo de compras. ¿Cómo gastan los turistas en España? Madrid : s.n., 2017.

Choi, M., Yoonjoung, C. y Law, R. 2016. Progress in shopping tourism. Hong Kong : Journal of Travel and Tourism Market, 2016.

Cifre, Y. 2017. Determinación de factores críticos de éxito para el desarrollo del Turismo de Compras en La Habana. Propuesta de acciones. La Habana : Trabajo de Diploma. Facultad de Turismo. Universidad de La Habana., 2017. 
Codling, S. 2000. Benchmarking. Madrid : Asociación Española de Normalización y Certificación, 2000.

Cubadebate. 2018. Cubadebate. Nota rectificada: Decrece en un 7\% llegada de turistas a Cuba en primer trimestre. [En línea] 2018. [Citado el: 30 de abril de 2018.] www.cubadebate.cu/noticias/2018/04/24/nota-rectificada-decrece-enun-7llegada-de-turistas-a-cuba-en-primer-trimestre/ .

Ecured. S/A. Ecured. América Latina. [En línea] S/A. [Citado el: 9 de febrero de 2018.] https://www.ecured.cu/america-latina/.

El Clarín. 2017. El Clarín. Rebajas de temporada 2017. [En línea] 2017. [Citado el: 5 de abril de 2018.] www.clarin.com/compras/tips/rebajas-de-temporada.

Ernst \& Young. 2015. La nueva era del retail: Impactos y retos del turismo de compras.

s.1. : Centro de Estudios de la Ernst \& Young S.L., 2015.

Estadística y censos. 2017. Estadística ciudad. Indicadores de turismo. [En línea] 2017. [Citado el: 5 de mayo de 2018.] www.estadisticaciudad.gob.ar/eyc/.

Excelsior. 2017. Excelsior. Calendario de rebajas 2017. [En línea] 2017. [Citado el: 5 de abril de 2018.] www.excelsior.com.mx/global/2017/11/21/1202711.

García, M. 2012. Diagnóstico de eficiencia en la gestión del comercio minorista de bienes en Villa Clara. Villa Clara : Trabajo de Diploma. Facultad de Ciencias Económicas. Universidad Central “Marta Abreu” de Las Villas, 2012.

García, R. 2016. Aprende de Turismo. El Turismo de Compras, una nueva motivación para viajar. [En línea] 2016. [Citado el: 1 de febrero de 2018.] http://www.aprendedeturismo.org/elturismo-de-comprasuna-nueva- motivacionpara-viajar/ .

Global Blue. 2012. Global Blue. The Globe Shopper City Index. Europe and Asia-Pacific. [En línea] 2012. [Citado el: 4 de febrero de 2018.] http://globeshopperindex.com/en/Download/.

Gobierno de la República de Cuba. S/A. Cubagob. Ministerio de Comercio Interior. [En línea] S/A. [Citado el: 3 de marzo de 2018.] http://www.cubagob.cu/des_eco/mincin/mincin.htm .

Goeldner, Ch., Cohen, B. y Brent, J. 2000. Tourism. Principles, Practices, Philosophies.

New York : John Whiley and Sons, Inc., 2000.

Hikersbay. 2018. Comparador de precios Hikersbay. [En línea] 2018. [Citado el: 4 de mayo de 2018.] www.hikersbay.com/america. 
Hosteltur. 2017. Revista Hosteltur. Turismo en Latinoamérica creció 7\% hasta abril. [En línea] 2017. [Citado el: 2 de febrero de 2018.] https://www.hosteltur.lat/148000_turismo-latinoamerica-crecio-7-abril.html.

—. 2018. Revista Hosteltur. Desempeño del turismo en Cuba. [En línea] 2018. [Citado el:

12 de febrerode 2018.] https://www.hosteltur.lat/desarrollo_del_turismo_en_cuba.html .

Ibáñez, R. y Cabrera, C. 2011. Teoría General del Turismo. Baja California : s.n., 2011. ICCA. 2017. ICCA World. ICCA Statistics Report Country \& City Rankings. [En línea]

2017. [Citado el: 8 de mayo de 2018.] www.iccaworld.org/dcps/doc.cfm.

ICSC Latinoamérica. 2017. International Council of Shopping Centers. Latin America. Mexican Shopping Center. [En línea] 2017. [Citado el: 1 de abril de 2018.] www.icsc.org/latam/news/mexican-shopping-centers.

Infotur. 2018. Información Turística de Cuba. La Habana. Transportación. [En línea] 2018. [Citado el: 6 de mayo de 2018.] http://www.infotur.cu/servicios.aspx.

Instituto Brasileño de Turismo . 2018. EMBRATUR. Información General. [En línea] 2018. [Citado el: 26 de marzo de 2018.] http://www.embratur.gov.br/.

Interbrand. 2017. Ranking de las marcas más valiosas del retail en América Latina. Nueva York : s.n., 2017.

Jansen-Verbeke, M. 1991. Leisure Sopping. A magic concept for the tourism industry?

Nijmegen : Tourism Management, 1991.

Kayak. 2017. Kayak. ¿Cuánto cuesta hospedarse en América Latina? [En línea] 2017. [Citado el: 26 de abril de 2018.] www.kayak.com.mx/cuanto-cuestahospedarseen- america-latina.

Kim, V. 2013. CNN. Las 12 mejores ciudades del mundo para ir de compras. . [En línea] 2013. [Citado el: 4 de febrero de 2018.] http://cnnespanol.cnn.com/2013/11/19/las- 12mejores-ciudades-del-mundoparair-de-compras/.

Kozak, M. 2004. Destination benchmarking. Concepts, practices and operations.

Cambridge : CABI Publishing, 2004. 
Lonely Planet. 2018. América. Atractivos. [En línea] 2018. [Citado el: 26 de mayo de 2018.] www.lonelyplanet.com.

López, L. 2010. Benchmarking y su aplicación al turismo. Santiago de Compostela : Revista de Estudios Politécnicos, 2010. Vol. VIII. 14.

Madrid, N. 2017. Pricetravel. Turismo de Compras en el mundo: 10 ciudades a elegir. [En línea] 2017. [Citado el: 4 de febrero de 2018.] http://revista.pricetravel.co/viaja- poreuropa/2017/01/09/turismo-de-compras/.

Manapocatl, E. 2016. Modalidades turísticas. Conceptos y tipologías. Baja California : s.n., 2016.

Martín, R. 2006. Principios, organización y práctica del turismo. La Habana : Félix Varela, 2006. Vol. 1.

Martín, V. 2015. Comercio y turismo. Dos caras de una misma moneda. Distribución y consumo. 2015, Vol. 2.

Merino, M. y Pérez, J. 2012. Definiciones. Comercio. [En línea] 2012. [Citado el: 15 de febrero de 2018.] http://definicion.de/comercio/.

Ministerio de Comercio Interior. 2015. Informe de los establecimientos comerciales de La Habana. La Habana : Gobierno de Cuba, 2015.

Montalvo, S. y Andrada, M. 2005. Investigación comercial y de mercados. Andalucía : Máster en comercio electrónico y marketing directo ICEMD, 2005.

NC ISO 9004. 2009. Gestión para el éxito sostenido de una organización. Enfoque de gestión de la calidad. La Habana : Oficina Nacional de Normalización, 2009.

—. 2015. Opciones. Llegará a Cuba la afamada marca de modas Chanel . [En línea] 2015. [Citado el: 20 de marzo de 2018.] http://www.opciones.cu/cuba/2015-1022/llegara-a-cuba-la-afamada-marca-demodas-chanel/ .

Organización Mundial del Turismo. 2014. Global Report on Shopping Tourism. Madrid : AM Reports, 2014. Vol. 8.

—. 2018. Organización Mundial del Turismo. Resultados del turismo internacional en 2017: los más altos en siete años. [En línea] 2018. [Citado el:

Organización Mundial del Turismo; Naciones Unidas. 2010. Recomendaciones internacionales para estadística de turismo 2008. Madrid : Departamento de Asuntos Económicos y Sociales., 2010.

Partido Comunista de Cuba. 2016. Lineamientos de la política económica y social del Partido y la Revolución. VII Congreso del Partido Comunista de Cuba. La Habana 
: s.n., 2016.

Perelló, J. 2018. Economía del turismo e importancia de la información para el análisis de expertos. La Habana : III Fórum provincial de periodismo económico ANECUPEC, 2018.

—. 2005. Introducción a la investigación de mercados turísticos. La Habana : Centro de Estudios Turísticos, 2005.

Rodríguez, J. 2018. Cubadebate. Cuba y su economía: El 2017 recién concluido y un 2018 que apenas comienza (IV). . [En línea] 2018. [Citado el: 10 de abril de 2018.] http://www.cubadebate.cu/opinion/2018/03/17/cuba-y-sueconomia-el-2017recien-concluido-y-un-2018-que-apenas-comienza-iv/ .

Safe Around. 2018. SafeAround. Americas. [En línea] 2018. [Citado el: 2 de mayo de 2018.] safearound.com/americas.

Sancho, A. y García, G. 2010. El posicionamiento de un destino turístico en un mercado globalizado y competitivo: Comparación de prácticas entre competidores y líderes. Valencia : s.n., 2010.

Timothy, D. 2005. Shopping tourism, retailing and leisure. Clevedon : Channel View Publications, 2005.

Trip Advisor. 2018. América Latina. Atractivos. [En línea] 2018. [Citado el: 28 de mayo de 2018.] www.tripadvisor.es.

—. 2018. América Latina. Centros Comerciales. [En línea] 2018. [Citado el: 26 de abril de 2018.] www.tripadvisor.es.

—. 2018. América Latina. Restaurantes. [En línea] 2018. [Citado el: 27 de mayo de 2018.] www.tripadvisor.es.

Turespaña. 2015. Plan de turismo de compras 2015. Madrid : s.n., 2015.

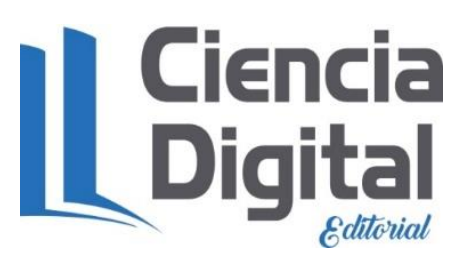




\section{PARA CITAR EL ARTÍCULO INDEXADO.}

Bulnes Mann, D. M. (2021). Turismo de compras: Retos, oportunidades y estrategias de desarrollo para el destino La Habana. Explorador Digital, 5(1), 365-388. https://doi.org/10.33262/exploradordigital.v5i1.1508

\section{Liencia}

El artículo que se publica es de exclusiva responsabilidad de los autores y no necesariamente reflejan el pensamiento de la Revista Explorador Digital.

El artículo queda en propiedad de la revista y, por tanto, su publicación parcial y/o total en otro medio tiene que ser autorizado por el director de la Revista Explorador Digital.
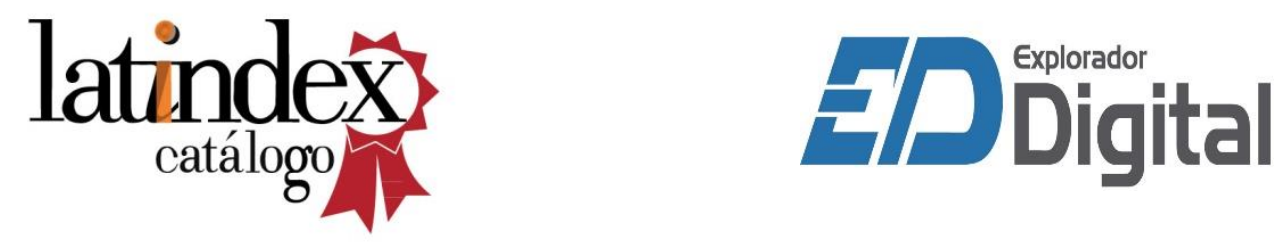